\title{
Bioactive Food Compounds As Epigenetic Regulators
}

\section{Compuestos Bioactivos de los Alimentos como Reguladores Epigenéticos}

\author{
Iván Enrique Naranjo Logroño', Anthony Alfonso Naranjo Coronel ${ }^{2}$, Ashley \\ Carolina Cuzco Macías ${ }^{1}$, and Alison Tamara Ruiz Chico ${ }^{1}$ \\ ${ }^{1}$ Carrera de Medicina, Facultad de Salud Pública, ESPOCH, Riobamba, Chimborazo, Ecuador \\ ${ }^{2}$ Médico Cirujano, COLPOMED Centro, Hospital del Día, Riobamba, Chimborazo, Ecuador
}

XVIII International Seminar on Health, Food and Human Nutrition

Corresponding Author:

Iván Enrique Naranjo Logroño

naranjometropolitana@hotmail.com

Published: 9 September 2021

Production and Hosting by

Knowledge E

(c) Iván Enrique Naranjo Logroño et al. This article is distributed under the terms of the Creative Commons Attribution License, which permits unrestricted use and redistribution provided that the original author and source are credited.
S OPEN ACCESS

\section{Abstract}

Introduction: Nutrigenomics explores and defines the rapidly evolving field of interactions in the diet and genome, through the use of nutrition, proteomics, physiology, biochemistry and epigenomics to seek and explain the mutual interactions between genes and nutrients from a molecular approach. Objective: To summarize the evidence of the impact that bioactive compounds of food exerts on epigenetic events aimed primarily at disease prevention. Methods: A literature review was carried out on the main bioactive compounds of food as epigenetic regulators, in the following search engines: COCHRANE, PUBMED, MEDLINE, LILACS, SCIELO, CINAHL, EBSCO and SCOPUS. The synthetic analytical method was used. Results: 40 bibliographic sources were found, of which 33 scientific articles were used that provided relevant information on the subject and seven articles were excluded. Discussion: There is scientific evidence about various bioactive compounds that experience efficacy in disease prevention through epigenetic regulation. Conclusion: With the continuous progress of changes in lifestyle, it is necessary to study new molecules, which can potentially be used for disease prevention and find new drugs that can be crucial in the treatment of different diseases.

Keywords: bioactive, food, regulators, epigenetics.

\section{Resumen}

Introducción: La nutrigenómica explora y define el campo en rápida evolución de las interacciones en la dieta y el genoma, mediante el uso de la nutrición, proteómica, fisiología, bioquímica y epigenómica para buscar y explicar las mutuas interacciones existentes entre genes y nutrientes desde un enfoque molecular. Objetivo: Resumir la evidencia del impacto que ejercen los compuestos bioactivos de los alimentos sobre los eventos epigenéticos dirigido principalmente en la prevención de enfermedades. Métodos: Se realizó una revisión bibliográfica acerca de los principales compuestos bioactivos de los alimentos como reguladores epigenéticos, en los siguientes buscadores: COCHRANE, PUBMED, MEDLINE, LILACS, SCIELO, CINAHL, EBSCO y SCOPUS. Se empleó el método analítico sintético. Resultados: Se encontraron 40 fuentes bibliográficas, de los cuales se utilizaron 33 artículos científicos que aportaron información relevante del tema y se excluyeron siete artículos. Discusión: Existe evidencia científica acerca de diversos compuestos bioactivos que demuestran eficacia en la prevención de enfermedades mediante la regulación epigenética. Conclusiones: Con el avance continuo de los cambios en el estilo de vida, es necesario estudiar 
nuevas moléculas, que pueden usarse potencialmente para la prevención de enfermedades y encontrar nuevos medicamentos que pueden ser cruciales en el tratamiento de distintas enfermedades.

Palabras Clave: bioactivos, alimentos, reguladores, epigenética.

\section{Introducción}

Desde la antigüedad, el ser humano ha conocido que el medio ambiente y los alimentos pueden influir con la condición de salud de un individuo y han usado alimentos y plantas como medicamentos. Con el avance de la ciencia, especialmente después de la conclusión del Proyecto del Genoma Humano, los científicos comenzaron a cuestionarse si la interacción entre los genes y los compuestos bioactivos de los alimentos podría influir positiva o negativamente en la salud de un individuo [1]. Para evaluar esta interacción entre genes y nutrientes, se creó el término 'Nutrigenómica', misma que explora y define el campo en rápida evolución de las interacciones de la dieta y el genoma, mediante el uso de la nutrición, proteómica, fisiología, bioquímica y epigenómica para buscar y explicar las mutuas interacciones existentes entre genes y nutrientes desde un enfoque molecular $[1,2]$.

A nivel molecular, los nutrientes transmiten señales que pueden traducirse en cambios en la expresión de genes, proteínas y metabolitos. La aplicación de la nutrigenómica a la vida cotidiana como el futuro de la ciencia de la nutrición ofrece nuevas herramientas para que se diseñen y receten dietas para las personas basado en su genoma y sus variaciones genéticas [3].

El descubrimiento de estas interacciones (gen-nutriente) ayudará a prescribir dietas personalizadas de acuerdo con el genotipo de cada individuo. Por lo tanto, será posible mitigar los síntomas de enfermedades existentes o prevenir enfermedades futuras, especialmente en el área de las Enfermedades Crónicas No Transmisibles, que actualmente se consideran un importante problema de salud pública mundial [1].

Las modificaciones de la impronta epigenética están relacionadas con el desarrollo natural y la diferenciación celular, así como con la progresión de enfermedades crónicas comunes. La plasticidad de estas marcas proporciona potencial para terapias de enfermedades y estrategias de prevención. Se ha demostrado que los macro y micronutrientes modulan el riesgo de enfermedad en parte a través de los efectos sobre el epigenoma [4].

Actualmente existe un gran interés por conocer y caracterizar qué alimentos tienen efectos en la regulación epigenética. Esta revisión tiene como objetivo resumir la evidencia del impacto que ejercen los compuestos bioactivos de los alimentos sobre los eventos epigenéticos dirigido principalmente en la prevención de enfermedades. 


\section{Metodología}

Se realizó una revisión bibliográfica acerca de los principales compuestos bioactivos de los alimentos como reguladores epigenéticos, en los siguientes buscadores: COCHRANE, PUBMED, MEDLINE, LILACS, SCIELO, CINAHL, EBSCO, SCIENCE DIRECT, SCOPUS; las palabras claves empleadas fueron: Bioactivos, alimentos, reguladores, epigenética, entre otros.

La investigación bibliográfica se realizó en el periodo comprendido entre octubre 2019 y enero 2020. Se empleó el método analítico sintético.

Dentro de los criterios de inclusión se encuentran ensayos clínicos controlados y randomizados con información referente a los principales compuestos bioactivos de los alimentos como reguladores epigenéticos, orientados en los idiomas de español e inglés, desde el año 2011 hasta el 2019. Se excluyeron aquellos que no cumplieron los criterios anteriormente mencionados.

\section{Resultados}

Como resultado de la búsqueda, se encontraron 40 fuentes bibliográficas referentes a los principales compuestos bioactivos de los alimentos como reguladores epigenéticos, de los cuales se utilizaron 33 artículos científicos que aportaron información relevante del tema y se excluyeron 7 artículos debido a que no cumplieron con los criterios de inclusión definidos.

\section{Discusión}

A continuación, se describen los principales compuestos bioactivos que han demostrado eficacia en la prevención de enfermedades mediante la regulación epigenética:

\subsection{Curcumina}

La curcumina (diferuloilmetano), un compuesto polifenólico, es un componente de Curcuma longa, que posee una gran cantidad de acciones biológicas, por lo que su uso se ha implicado en diversas enfermedades, incluyendo cáncer, enfermedades respiratorias, trastornos metabólicos y trastornos neurodegenerativos y recientemente se ha demostrado que modula varias enfermedades a través de la regulación epigenética, sin ningún cambio en la secuencia de ADN [5-7], resultando en un importante mecanismo por el cual este componente de la dieta puede activar o desactivar selectivamente la expresión génica [8]. Las funciones reguladoras epigenéticas de la curcumina incluyen: Regulación de los cambios de histonas a través de la regulación de histonas acetiltransferasas (HAT) e histona desacetilasas (HDAC); inhibición de las metiltransferasas de ADN (DNMT), que ha sido bien definida a partir de estudios recientes sobre su función como agente hipometilante de $A D N$; y regulación de micro $A R N$ (miARN) que 
son pequeños ARN no codificantes que están comúnmente presentes en las células normales y tumorales [7]. Además, la curcumina puede unir directamente y alterar múltiples cascadas de señalización celular, que se pueden aprovechar para combatir patologías seleccionadas. La investigación en esta área proveerá más información sobre el uso de la curcumina y sus análogos como agentes eficaces para atacar diferentes enfermedades en el futuro [9].

\subsection{Polifenoles}

Los polifenoles son micronutrientes potentes que se pueden encontrar en distintas fuentes de alimentos y especias, juegan un papel transcendental basado en nutrientes en la prevención de diversos padecimientos [10]. Cuantiosos estudios han indicado que poseen propiedades de modificación de los mecanismos epigenéticos que modulan la expresión génica. Estos polifenoles, cuando se administran de forma dependiente de la dosis y combinatoria, pueden tener un efecto mejorado sobre los cambios epigenéticos, que desempeñan un papel crucial en la prevención y terapia de enfermedades, como el cáncer. Clásicamente, la investigación del cáncer se centraba principalmente en los cambios genéticos, aclarando la sobreexpresión/mutación de los oncogenes y la inactivación de los genes supresores de tumores [11]. Sin embargo, los polifenoles pueden prevenir el cáncer al modular las aberraciones epigenéticas que tienen lugar en la metilación del ADN, las modificaciones de histonas y los miARN. Al alterar estas epimutaciones, regulan la dinámica de la cromatina y la expresión de genes que inducen o suprimen la formación de cáncer [11].

Recientemente, los cambios epigenéticos en los patrones de metilación del ADN en los sitios CPG (epimutaciones) o los estados de cromatina desregulados de los genes promotores de tumores y los ARN no codificantes surgieron como los principales factores rectores en la progresión tumoral y la sensibilidad a los medicamentos contra el cáncer. Dado que las marcas epigenéticas son reversibles en contraste con los defectos genéticos, los polifenoles nutricionales quimiopreventivos se evalúan actualmente por su capacidad para revertir las marcas epigenéticas adversas en las células cancerosas para atenuar la progresión de la tumorigénesis, prevenir metástasis o sensibilizar sobre la sensibilidad a los medicamentos [12].

\subsection{Sulforafano (isotiocianatos)}

El sulforafano (SFN) es un fitoquímico derivado de vegetales crucíferos, como el brócoli y la coliflor, que tiene múltiples objetivos moleculares y acción anticancerígena [5], siendo uno de los inhibidores de histona desacetilasa más potentes hasta la fecha [13]. La evidencia emergente indica que el SFN altera los mecanismos epigenéticos [5]. Los mecanismos epigenéticos presentes en las células tumorales comprenden: La modificación de histonas posterior a la traducción, es decir desacetilación y metilación; regulación postranscripcional de la expresión génica por miARN no codificante; hipometilación global de ADN; y promotor de la hipermetilación de genes supresores 
de tumores y genes importantes para la regulación del ciclo celular, la diferenciación celular y la apoptosis [14].

EI SFN ejerce sus efectos anticancerígenos al modular las vías de señalización clave y los genes involucrados en la inducción de apoptosis, la detención del ciclo celular y la inhibición de la angiogénesis [15].

\subsection{Quercetina}

La quercetina forma parte de la familia flavonoide, se encuentra presente en diversas frutas y verduras, como manzanas y cebollas, respectivamente [16]. La evidencia actual sugiere que la quercetina y otros flavonoides pueden contrarrestar la regulación epigenética adversa implicada en diversas enfermedades como el cáncer [16].

En estudios recientes se evidenció que la apoptosis mejorada, inducida por la quercetina, podría ser causada en parte, por la desmetilación del ADN, por la inhibición de HDAC y por el enriquecimiento de $\mathrm{H} 3 \mathrm{ac}$ y $\mathrm{H} 4 \mathrm{ac}$ en las regiones promotoras de genes involucrados en la vía de apoptosis, lo que lleva a su activación de transcripción [17]. En las células tumorales, la quercetina se ha observado que bloquea el ciclo celular e induce efectos pro-apoptóticos sin afectar las células normales. Por otra parte, Tan et al., demostró que la quercetina inhibe el crecimiento tumoral mediante la activación de p16INK4a inducida por la desmetilación del promotor en células del cáncer colorrectal (CCR), también que las combinaciones de quercetina y curcumina restauran los niveles de proteínas en células de cáncer de próstata negativas para los receptores de andrógenos. Estos efectos fueron mediados por la disminución de DNMT, resultando en hipometilación global e inducción de apoptosis a través de la despolarización mitocondrial [18].

También se ha evidenciado los efectos sinérgicos del tratamiento combinado de quercetina y curcumina resultaron en la sensibilización de las células de cáncer de próstata resistentes al tratamiento antiandrogénico [18].

\subsection{Flavonas}

La manzanilla, el apio y el perejil son fuentes ricas en apigenina; el aceite de oliva, las zanahorias, pimientos, apio, menta, tomillo, romero y orégano son importantes fuentes dietéticas de luteolina. La apigenina y la luteolina ejercen efectos inhibitorios sobre la 5-citosina DNMT como se muestra, utilizando extractos nucleares de células KYSE-510. En un estudio, ambas flavonas se probaron a concentraciones de 20 y $50 \mu \mathrm{mol} / \mathrm{L}$. La luteolina mostró una inhibición más pronunciada de las enzimas DNMT con una eficacia de aproximadamente el $50 \%$ a $50 \mu \mathrm{mol} / \mathrm{L}$, mientras que la apigenina solo mostró una inhibición del 35\% a $50 \mu \mathrm{mol} / \mathrm{L}$. La apigenina causa la detención del ciclo celular y la apoptosis en las células de cáncer de próstata humano. Una investigación reveló que la inhibición del crecimiento mediada por apigenina se debe a la inhibición de la HDAC de clase I. Los autores demostraron que el tratamiento de las células PC-3 y 22Rv1 con 20-40 $\mu \mathrm{mol} / \mathrm{L}$ de apigenina inhibió HDAC1 y HDAC3 en los niveles de ARNm y proteínas, lo que resultó en una hiperacetilación global de histonas H3 y H4 [19]. 


\subsection{Genisteína}

La genisteína (4', 5, 7-trihidroxiisoflavona) es una isoflavona fitoestrógeno que se encuentra en las plantas de Leguminoseae como la soya y en los productos de soya [20]. Existe evidencia acumulada que muestra que el consumo de genisteína en adultos afecta los procesos epigenéticos. Los efectos biológicos de su consumo en la etapa temprana de vida son controvertidos e inconclusos [20].

Pocos estudios han investigado el impacto del consumo temprano de isoflavonas de soja en la función reproductiva en la edad adulta. Una investigación reciente realizada en el contexto del estudio de Alimentación Infantil y Desarrollo Temprano mostró que, en comparación con las bebés alimentadas con fórmula de vaca, las alimentadas con fórmula de soya, que contienen niveles significativos de isoflavonas de soja, durante los primeros 9 meses de vida presentaron diferencias en la metilación del ADN en el gen rico en prolina 5 como (PRR5L). Sin embargo, la importancia de dicha alteración epigenética no está clara en el contexto de las células epiteliales vaginales, ni su impacto potencial en la salud reproductiva femenina [20].

Se ha propuesto que la ingesta temprana de soja podría representar un aspecto clave que afecta sus efectos protectores propuestos contra el cáncer de mama. Además, la genisteína podría ejercer sus efectos protectores al apuntar epigenéticamente a la expresión génica en las células madre mamarias, una hipótesis que aún debe confirmarse [20].

\subsection{Butirato}

El butirato es un ácido graso de cadena corta (AGCC), producto de la fermentación de fibras dietéticas en el intestino grueso [21]. Puede alterar las expresiones genéticas y epigenéticas. La expresión aberrante de miARN está asociada con la iniciación, progresión y metástasis del CCR. Además, el butirato puede modular la proliferación celular, la diferenciación, la apoptosis y la expresión de miARN en CCR [22].

Las bacterias que habitan en el epitelio del tracto digestivo proporcionan las vías bioquímicas esenciales para la fermentación de fibras dietéticas que de otro modo no serían asimilables, lo que lleva a la producción de AGCC. De los principales AGCC, el butirato ha recibido una atención particular debido a sus numerosos efectos provechosos sobre la salud del tracto intestinal y los tejidos periféricos. Los mecanismos de acción de este ácido orgánico son diferentes. Muchos de estos están relacionados con su potente efecto regulador sobre la expresión génica, ya que el butirato es un inhibidor de la HDAC que desempeña un papel predominante en la regulación epigenética de la expresión génica y la función celular [23]

\subsection{Selenio}

El selenio (Se) es un nutriente que se encuentra en las nueces de Brasil, el pollo, la carne de caza y la carne de res. Otras formas químicas de Se incluyen selemetionina, selenocisteína, selenato y selenito. Él Se es un elemento esencial con propiedades 
antioxidantes, proapoptóticas, reparadoras de ADN y anticancerígenas. Además, varias otras selenoproteínas (es decir, proteína de unión a selenio-1) se han indicado como importantes en el desarrollo de enfermedades como distintos tipos de cánceres; sin embargo, sus efectos epigenéticos no se han definido claramente [24].

El progreso reciente sugiere funciones importantes de ciertas selenoproteínas en la regulación epigenética de la metilación del promotor, modificaciones de histonas, expresiones de ARN no codificantes y estabilidad del genoma. Por el contrario, tales eventos epigenéticos también pueden influir en la expresión de selenoproteína [24].

En un ensayo diseñado para evaluar él Se en cánceres de piel no melanoma, en individuos con alto riesgo de desarrollar esta enfermedad recibieron $200 \mu \mathrm{g}$ de Se o placebo por vía oral por día durante un promedio de 4,5 años. Este ensayo no previno el cáncer de piel, pero produjo una disminución secundaria significativa del $44 \%$ en la incidencia de cáncer de pulmón. Se ha relacionado con la metilación del ADN en modelos celulares y animales y Xiang et al., descubrieron que los tratamientos con Se causaron la desmetilación parcial del ADN del promotor y la reexpresión de GSTP1 en las células de cáncer de próstata. Además, se ha demostrado que la deficiencia de Se causa hipometilación global y metilación del promotor de los genes supresores de tumores p16 y p53. Además, las investigaciones han demostrado que el tratamiento de las células de cáncer de próstata con selenito, una forma inorgánica de ser, puede restablecer la expresión de genes anticancerígenos silenciados por hipermetilación, lo que sugiere que la regulación epigenética por Se puede desempeñar un papel en la prevención del cáncer, y también puede alterar la metilación global del ADN en el hígado. Aunque estos estudios son interesantes, se necesitan más estudios que involucren la influencia epigenética del selenio para apreciar completamente el impacto del selenio en el epigenoma [25-27].

\subsection{Inositol}

Se encuentra en ciertos alimentos como la lecitina, el hígado de ternera, el germen de trigo, las nueces, la avena, legumbres, etc. El inositol es el segundo sistema de mensajería, responsable del sistema de señalización metabólico y celular. El mioinositol es una forma de inositol, que afecta algunas enzimas involucradas en el metabolismo energético, la lipogénesis, aumentando la actividad de las proteínas transportadoras de glucosa. Además, el fosfotidil inositol participa en la comunicación transmembrana. El Inositol 1-4-5 trifosfato (IP3) proporciona movilización intracelular de calcio [28]. Los estudios indican que la inactivación epigenética del polifosfato de inositol 4-fosfatasa $B$ (INPP4B), un regulador de la vía de señalización PI3K/AKT en el carcinoma nasofaríngeo (CNF) asociado al virus de Epstein-Bar (VEB), constituye uno de los mecanismos clave para activar la cascada de señalización de PI3K/AKT y desempeñar un papel en la tumorigénesis de CNF [28]. 


\subsection{Folato}

El folato es una vitamina esencial soluble en agua que ocurre naturalmente en alimentos seleccionados, así como en la forma sintética (ácido fólico) utilizada en suplementos y en programas de enriquecimiento de alimentos [29].

La metilación del ADN es una modificación epigenética crítica para la regulación y el desarrollo del genoma normal. La vitamina folato es una fuente clave del grupo de carbono utilizado para metilar el ADN. Debido a que el desarrollo normal de los mamíferos depende de la metilación del ADN, existe un enorme interés en evaluar el potencial de cambios en la ingesta de folato para modular la metilación del ADN como un biomarcador para el estado del folato y como un vínculo mecanístico para los trastornos del desarrollo y las enfermedades crónicas [29].

Varios estudios han informado que la ingesta adecuada de folato puede conferir un efecto protector para el riesgo de CCR. Sin embargo, el aumento en la ingesta de folato en muchas poblaciones ha generado algunas preocupaciones sobre los posibles efectos nocivos con respecto al crecimiento tumoral, pero hasta ahora no ha habido informes consistentes de un mayor riesgo de CCR debido a la suplementación con folato. Sin embargo, los estudios en animales han sugerido un efecto modulador de la ingesta de folato dependiendo del momento de la carcinogénesis. Los cambios epigenéticos, incluidas las alteraciones en la metilación del ADN, se han estudiado ampliamente en CCR y se ha demostrado que ocurren en las primeras etapas de la tumorigénesis. En consecuencia, los cambios en la metilación del ADN podrían ser biomarcadores potenciales para el CCR y pueden ser útiles para el diagnóstico temprano, la terapia personalizada o el pronóstico [30, 31].

\subsection{Alil mercaptano}

Se ha demostrado que los derivados alílicos del ajo inducen la acetilación de histonas en varias células cancerosas humanas. El derivado de alilo más potente con respecto a la inhibición de HDAC es el alil mercaptano, que ejerce sus propiedades anticancerígenas in vitro a través de la hiperacetilación de CDKN1A, que posteriormente aumenta la expresión del gen CDKN1A y promueve la detención del ciclo celular. En estudios preclínicos, los mecanismos de acción informados de los compuestos derivados del ajo para la prevención y el tratamiento del cáncer son mucho más diversos y van desde la inducción de apoptosis y autofagia hasta la inhibición del angiogénesis y la proliferación. Un ensayo aleatorio de alimentación cruzada en humanos demostró que una sola comida de ajo crudo y triturado influye en la expresión de múltiples genes relacionados con la inmunidad y el cáncer, lo que sugiere que la bioactividad del ajo es multifacética. Sin embargo, en un estudio aleatorizado, doble ciego de intervención clínica, siete años de suplementación con ajo no redujeron la incidencia de lesiones gástricas precancerosas o cáncer gástrico en sujetos con alto riesgo de cáncer gástrico. Potencialmente, esto podría explicarse porque el grupo de población ya tenía un alto riesgo de cáncer gástrico, pero la utilidad generalizada de la suplementación con ajo 
probablemente no podrá utilizarse hasta que los mecanismos de acción se entiendan más completamente [32].

La creciente necesidad del uso de nutrición personalizada en pacientes está aumentando y se están realizando investigaciones sobre sus posibles efectos [33].

\section{Conclusiones}

Con el avance continuo de los cambios en el estilo de vida, es necesario estudiar nuevas moléculas presentes en los alimentos, que pueden usarse potencialmente para la prevención de enfermedades y encontrar nuevos medicamentos que pueden ser cruciales en el tratamiento de distintas enfermedades. Sin embargo, se requiere más investigación para establecer qué papel juegan exactamente los cambios epigenéticos en la mediación de los cambios observados en la expresión de genes y proteínas, y la relevancia de estos cambios para la salud.

\section{Agradecimiento}

Nuestro agradecimiento a la Escuela Superior Politécnica de Chimborazo, a la Facultad de Salud Pública y al XVIII Seminario Internacional de Salud, Alimentación y Nutrición Humana, a su comité organizador por su esfuerzo colaborativo en la difusión de investigación científica.

\section{Conflictos de Interés}

Los autores declaramos no tener ningún conflicto de interés.

\section{Limitaciones de Responsabilidad}

Los autores declaramos que toda la información recopilada en el presente documento es de nuestra entera responsabilidad y no de la institución en la que laboramos.

\section{Fuentes de Financiación}

La financiación de la presente investigación proviene de los mismos autores.

\section{References}

[1] Sales NMR, Pelegrini PB, Goersch MC. Nutrigenomics: Definitions and advances of this new science. J Clin Nutr Metab. 2014.

[2] Vahid F, Zand H, Nosrat-Mirshekarlou E, Najafi R, Hekmatdoost A. The role dietary of bioactive compounds on the regulation of histone acetylases and deacetylases: A review. Gene Rep. Elsevier. 2015;562:8-15.

[3] Dennett C. The Future of Nutrigenomics. Today's Dietit. 2017;19. 
[4] Speckmann B, Grune T. Epigenetic effects of selenium and their implications for health. Epigenetics. 2015;10(3):179-90.

[5] Atwell LL, Beaver LM, Shannon J, Williams DE, Dashwood RH, Ho E. Epigenetic regulation by Sulforaphane: Opportunities for breast and prostate cancer chemoprevention. Curr Pharmacol Rep. 2015;1(2):102-11.

[6] Pandey A, Kulkarni YA, Gaikwad AB. Fruits, vegetables, and herbs: Bioactive foods in health promotion. Elsevier Inc.; 2016. Curcumin: The epigenetic therapy; p. 105-19.

[7] Boyanapalli SSS, Kong ANT. "Curcumin, the king of spices": Epigenetic regulatory mechanisms in the prevention of cancer, neurological, and inflammatory diseases. Curr Pharmacol Rep. 2015;1(2):129-39.

[8] Reuter S, Gupta SC, Park B, Goel A, Aggarwal BB. Epigenetic changes induced by curcumin and other natural compounds. Genes Nutr. 2011;6(2):93-108.

[9] Azadand RSGK. Epigenetics of Curcumin: A Gifted Dietary Therapeutics Compound. J Carcinog Mutagen. 2015;06(01):1-6.

[10] Arora I, Sharma M, Tollefsbol TO. Combinatorial epigenetics impact of polyphenols and phytochemicals in cancer prevention and therapy. Int J Mol Cell Med. MDPI AG. 2019;20.

[11] Bag A, Bag N. Tea polyphenols and prevention of epigenetic aberrations in cancer. J Nat Sci Biol Med. 2018;9(1):2-5.

[12] Vanden BW. Epigenetic impact of dietary polyphenols in cancer chemoprevention: Lifelong remodeling of our epigenomes. harmacognosy Res. 2012;65(6):565-76.

[13] Martin SL, Kala R, Tollefsbol TO. Mechanisms for the inhibition of colon cancer cells by sulforaphane through epigenetic modulation of MicroRNA-21 and human telomerase reverse Transcriptase (hTERT) Down-regulation. Curr Cancer Drug Targets. 2017;18(1).

[14] Kaufman-Szymczyk A, Majewski G, Lubecka-Pietruszewska K, Fabianowska-Majewska K. The role of sulforaphane in epigenetic mechanisms, including interdependence between histone modification and DNA methylation. Int J Mol Med Sci. 2015;16(12):29732-43.

[15] Su X, Jiang X, Meng L, Dong X, Shen Y, Xin Y. Anticancer activity of Sulforaphane: The epigenetic mechanisms and the Nrf2 signaling pathway. Oxid Med Cell Longev. 2018:1-10.

[16] Russo GL, Ungaro P. Epigenetics of Cancer Prevention. Elsevier; 2019. Epigenetic Mechanisms of Quercetin and other flavonoids in cancer therapy and prevention; p. 187-202.

[17] Alvarez MC, Maso V, Torello CO, Ferro KP, Saad STO. The polyphenol quercetin induces cell death in leukemia by targeting epigenetic regulators of pro-apoptotic genes. J Clin Epigenet. 2018;10(1):139.

[18] Carlos-Reyes Á, López-González JS, Meneses-Flores M, et al. Dietary compounds as epigenetic modulating agents in cancer. Mol Front J. 2019;10(Mar).

[19] Busch C, Burkard M, Leischner C, Lauer UM, Frank J, Venturelli S. Epigenetic activities of flavonoids in the prevention and treatment of cancer. J Clin Epigenet. 2015;7(1).

[20] Silva LBAR, Pinheiro-Castro N, Novaes GM, Pascoal G de FL, Ong TP. Bioactive food compounds, epigenetics and chronic disease prevention: Focus on early-life interventions with polyphenols. Food Res Int. 2019;125.

[21] Berni CR, Di Costanzo M, Leone L. The epigenetic effects of butyrate: Potential therapeutic implications for clinical practice. J Clin Epigenet. 2012;4(1)

[22] Bishop KS, Xu H, Marlow G. Epigenetic regulation of gene expression induced by butyrate in colorectal cancer: Involvement of microRNA. Genet Epigenet. 2017;9.

[23] Terova G, Díaz N, Rimoldi S, Ceccotti C, Gliozheni E, Piferrer F. Effects of Sodium Butyrate Treatment on histone modifications and the expression of genes related to Epigenetic regulatory mechanisms and immune response in European Sea Bass (Dicentrarchus Labrax) fed a plant-based diet. PLoS One. 2016;11(7).

[24] Lu H-Y, Somuncu B, Zhu J, Muftuoglu M, Cheng W-H. Handbook of Nutrition, Diet, and Epigenetics. Springer International Publishing; 2017. Selenoproteins and Epigenetic Regulation in Mammals; p. 1-15.

[25] Hardy TM, Tollefsbol TO. Epigenetic diet: Impact on the epigenome and cancer. Epigenomics. 2011;3(4):503-18.

[26] Jabłońska E, Reszka E. Selenium and epigenetics in cancer: Focus on DNA methylation. Nat Cancer. Academic Press Inc. 2017;136:193-234.

[27] Bermingham EN, Bassett SA, Young W, et al. Post-weaning selenium and folate supplementation affects gene and protein expression and global DNA methylation in mice fed high-fat diets. BMC Med Genomics. 2013;6.

[28] Celik E, Sanlier N. Effects of nutrient and bioactive food components on Alzheimer's disease and epigenetic. Crit Rev Food Sci Nutr. 2019;59(1):102-13.

[29] Crider KS, Yang TP, Berry RJ, Bailey LB. Folate and DNA Methylation: A review of molecular mechanisms and the evidence for Folate's Role. Adv Nutr. 2012;3(1):21-38. 
[30] Kok DE, Steegenga WT, McKay JA. Folate and epigenetics: Why we should not forget bacterial biosynthesis. Epigenomics. 2018;10(9):1147-50.

[31] Lévesque N, Leclerc D, Rozen R. Br J Cancer. Springer International Publishing; 2017. Folate and epigenetics: Colorectal cancer risk and detection; p. 1-19.

[32] Montgomery M, Srinivasan A. Epigenetic gene regulation by dietary compounds in cancer prevention. Adv Nutr. 2019;10(6):1012-28.

[33] Pavlidis C, Patrinos GP, Katsila T. Nutrigenomics: A controversy. Appl Transl Genom. 2015;4:50-3. 